\title{
Crotoxin induces apoptosis and autophagy in human lung carcinoma cells in vitro via activation of the p38 MAPK signaling pathway
}

\author{
Rong HAN", Hui LIANG\#, Zheng-hong QIN, Chun-yu LIU* \\ College of Pharmaceutical Science, Soochow University, Suzhou 215123, China
}

\begin{abstract}
Aim: Crotoxin (CrTX) is the primary toxin in South American rattlesnake (Crotalus durissus terrificus) venom, and exhibits antitumor and other pharmacological actions in vivo and in vitro. Here, we investigated the molecular mechanisms of the antitumor action of CrTX in human lung carcinoma cells in vitro.

Methods: Human lung squamous carcinoma SK-MES-1 cells were tested. The cytotoxicity of CrTX was evaluated in both MTT and colony formation assays. Cell cycle was investigated with flow cytometry. Cell apoptosis was studied with Hoechst 33258 and Annexin V-FITC staining. The levels of relevant proteins were analyzed using Western blot assays.

Results: $\operatorname{CrTX}(25,50,100 \mu \mathrm{mol} / \mathrm{L})$ inhibited the growth and colony formation of SK-MES-1 cells in dose- and time-dependent manners. CrTX increased the proportion of S phase cells and dose-dependently induced cell apoptosis, accompanied by down-regulating the expression of proliferating cell nuclear antigen (PCNA), and increasing the level of cleaved caspase-3. Furthermore, CrTX dosedependently increased the expression of autophagy-related proteins LC3-II and beclin 1, and decreased the level of p62 in the cells. Moreover, CrTX (50 $\mathrm{mmol} / \mathrm{L})$ significantly increased p38MAPK phosphorylation in the cells. Pretreatment of the cells with SB203580, a specific inhibitor of p38MAPK, blocked the inhibition of CrTX on cell proliferation, as well as CrTX-induced apoptosis and cleaved
\end{abstract} caspase-3 expression.

Conclusion: The p38MAPK signaling pathway mediates CrTX-induced apoptosis and autophagy of human lung carcinoma SK-MES-1 cells in vitro.

Keywords: human lung carcinoma; snake venom; crotoxin; cell cycle arrest; apoptosis; autophagy; caspase-3; proliferating cell nuclear antigen; p38 MAPK; SB203580

Acta Pharmacologica Sinica (2014) 35: 1323-1332; doi: 10.1038/aps.2014.62; published online 18 Aug 2014

\section{Introduction}

Lung cancer is the leading cause of cancer-related mortality worldwide $^{[1]}$. Non-small-cell lung cancer (NSCLC) accounts for approximately $85 \%$ of all cases of lung cancer, and the subtypes include adenocarcinoma, squamous carcinoma, and large cell carcinoma ${ }^{[2]}$. As most patients have advanced unresectable disease at the time of diagnosis, the treatment of choice is chemotherapy concurrent with chest radiation therapy; this combination therapy leads to a higher rate of survival. However, prolonged clinical success has been limited due to adverse side effects of available treatments. Many recent studies have focused on developing novel agents for

\footnotetext{
\#The two authors contributed equally to this work.

* To whom correspondence should be addressed.

E-mail liuchunyu@suda.edu.cn

Received 2014-03-09 Accepted 2014-06-05
}

the treatment of lung cancer.

Crotoxin (CrTX) is the major toxin in the venom of the South American rattlesnake Crotalus durissus terrificus; the toxin includes a weakly toxic basic Phospholipase A2 (CB) and a non-enzymatic, non-toxic acidic component (crotapotin, CA $)^{[3,4]}$. Various studies have shown that CrTX exhibits antitumor activity and other pharmacological actions in vivo and in vitro ${ }^{[5]}$. CrTX inhibits the growth of several tumor cell lines, including leukemia, lung, colon, renal, ovary, esophagus and mammary ductal carcinoma, melanoma and brain tumor cell lines ${ }^{[6-13]}$. Newman and Cura et al demonstrated that daily im injections of CrTX inhibited the growth of Lewis lung carcinoma and MX-1 human mammary carcinoma by $83 \%$ and $69 \%$, respectively ${ }^{[8,11]}$. Yan et al demonstrated that CrTX causes the collapse of the mitochondrial membrane potential, the release of cytochrome $c$ and the activation of caspase- 3 in the K562 chronic myeloid leukemia cell line ${ }^{[12]}$. CrTX inhib- 
its the growth of subcutaneously transplanted Eca-109 cells in nude mice via the induction of apoptosis and $G_{1}$ block $^{[13]}$. These results indicate that CrTX might be an appropriate candidate for cancer therapy.

Intracellular mitogen-activated protein kinases (MAPKs) are a group of protein serine/threonine kinases, including four distinct subgroups: (1) extracellular signal-regulated kinases (ERKs), (2) c-jun N-terminal or stress-activated protein kinases (JNK/SAPK), (3) ERK/big MAP kinase 1 (BMK1), and (4) the p38 group of protein kinases ${ }^{[14,15]}$. The four splice variants $(\alpha, \beta, \gamma$, and $\delta$ ) of the p38 group of MAPKs serve as a nexus for signal transduction and play a vital role in numerous biological processes ${ }^{[16]}$. p38 functions in a cell context-specific and cell type-specific manner to integrate signals that affect proliferation, differentiation, survival and cell migration ${ }^{[17]}$. It has been established that the p38 pathway is associated with a variety of antitumor activities, including apoptosis, cell cycle arrest and autophagic death ${ }^{[18-20]}$.

The effect of p38 is dependent not only on the stimuli but also on the cellular context ${ }^{[21]}$. p38MAPK may induce tumor cell $G_{1} / S$ or $G_{2} / M$ phase arrest, causing growth inhibition, apoptosis induction or cellular senescence, and may induce tumor cell autophagy, resulting in autophagic death ${ }^{[22,19]}$. Zhan et al showed that p38MAP kinase played a role in the switch from autophagy to apoptosis in MS-275-induced human colon cancer cells. The high expression of p38 induced cell autophagy, but low expression resulted in apoptosis ${ }^{[18]}$. Neoh et al showed that the induction of apoptosis by sinulariolide from soft coral is through mitochondrial-related and p38MAPK pathways in human bladder carcinoma cells. The inhibition of p38MAPK activity rescued the cell cytotoxicity of sinulariolide in TSGH cells ${ }^{[23]}$. However, many studies found that MAPK-mediated biological effects in NSCLC are complex. The p38 pathway plays a role in the development of lung cancer, but the activation of p38 in NSCLC can mediate apoptosis. We have recently reported that CrTX-induced cell apoptosis in human lung adenocarcinoma A549 cells by activating p38MAPK and caspase-3. We have also demonstrated CrTXinduced cell cycle arrest mediated by increased wt p53 expression and p-JNK up-regulation in SK-MES-1 cells ${ }^{[24,25]}$. However, the molecular mechanisms involved in the inhibition of SK-MES- 1 cells, specifically the effect of p38 on the induction of apoptosis and autophagy, are not well characterized. In this study, we further investigate the role of p38MAPK in CrTXinduced apoptosis and autophagy in human lung squamous carcinoma SK-MES-1 cells.

\section{Materials and methods Reagents}

CrTX was supplied by Celtic Biotech Ltd (Dublin, Ireland). It was purified from Crotalus durissus terrificus venom by a combination of size exclusion and anion exchange. The identity of the protein was confirmed through molecular weight determination by mass spectrometry (showing averaged signals at $9500 \mathrm{Da}$ and $14500 \mathrm{Da}$, in addition to the presence of isoforms). The purity, as determined by PAGE and size exclusion, was confirmed to be $>99 \%$. Lethality in mice was determined by the ip injection of $0.1 \mathrm{mg}$ of CrTX followed by monitoring for death within $3 \mathrm{~h}^{[25]}$. The p38 inhibitor SB203580 was purchased from Calbiochem, Inc (Madison, WI, USA).

\section{Cell culture}

The human Caucasian lung squamous carcinoma cell line SK-MES-1 was obtained from the Cell Bank of the Shanghai Institute of Biochemistry and Cell Biology, Chinese Academy of Sciences (Shanghai, China). The cells were cultured in a nutrient medium (RPMI-1640) (GIBCO Invitrogen, Grand Island, NY, USA) supplemented with heat-inactivated $10 \%$ fetal bovine serum (GIBCO Invitrogen, Grand Island, NY, USA), $100 \mathrm{U} / \mathrm{mL}$ penicillin, and $100 \mathrm{mg} / \mathrm{mL}$ streptomycin in a water-saturated atmosphere at $5 \% \mathrm{CO}_{2}$ and $37^{\circ} \mathrm{C}$. The culture medium was changed every 2 to $3 \mathrm{~d}$.

\section{Cell viability assay}

Cell viability was measured using the MTT assay. SK-MES-1 cells were plated in 96-well plates at a cell density of 50000 cells per well in $100 \mu \mathrm{L}$ of RPMI-1640 and allowed to grow in a $\mathrm{CO}_{2}$ incubator for $24 \mathrm{~h}$. The medium was then removed and replaced with fresh medium containing varying concentrations of CrTX (from 25 to $100 \mu \mathrm{g} / \mathrm{mL}$ ) for 24,48 , and $72 \mathrm{~h}$. The cultures were then incubated in $100 \mu \mathrm{L}$ of medium with $10 \mu \mathrm{L}$ of $5 \mathrm{mg} / \mathrm{mL}$ MTT solution for $3 \mathrm{~h}$ at $37^{\circ} \mathrm{C}$. The medium with MTT was removed, and $100 \mu \mathrm{L}$ of DMSO was added to each well to dissolve the formazan. The absorbance was read at 570 $\mathrm{nm}(630 \mathrm{~nm}$ as a reference) on a microplate reader (BIORADBenchmark, California, USA).

\section{Colony formation assay}

SK-MES-1 cells were seeded in six-well plates at a density of 500 cells per well for $24 \mathrm{~h}$. After treatment with varying concentrations of CrTX $(0,25,50$, or $100 \mu \mathrm{g} / \mathrm{mL})$ for $48 \mathrm{~h}$, the cells were cultured in normal medium for 2 weeks. The culture medium was changed every 2 to $3 \mathrm{~d}$. The medium was then discarded, and each well was washed twice with PBS. The colonies were fixed in methanol for $30 \mathrm{~min}$ and then stained with Giemsa. The number of colonies with $\geq 50$ cells was counted, and the colony forming efficiency was calculated (Percentage of colonies $=$ Number of colonies formed/Number of cells inoculated $\times 100 \%$ ). Experiments were repeated three times.

\section{Flow cytometry analysis of cell cycle and apoptosis}

SK-MES-1 cells were seeded into 50-mL culture flasks, and after treatment with $0,25,50$, or $100 \mu \mathrm{g} / \mathrm{ml}$ CrTX for $48 \mathrm{~h}$, cell cycle status and cell apoptosis were detected using the propidium iodide (PI) and Annexin V-FITC/PI assay by flow cytometry. Briefly, the cells were trypsinized, washed twice with ice-cooled phosphate buffered saline (PBS) and fixed with 70\% $\mathrm{EtOH}$. After overnight refrigeration at $4^{\circ} \mathrm{C}$ and subsequent rehydration in PBS for $30 \mathrm{~min}$ at $4^{\circ} \mathrm{C}$, the cells were stained at $37^{\circ} \mathrm{C}$ for $20 \mathrm{~min}$ with $50 \mu \mathrm{g} / \mathrm{mL}$ propidium iodide and 100 units/mL RNase. Analyses were performed with the aid of an 
EPICS XL flow cytometer (Beckman Coulter, Miami, FL, USA) equipped with the Multicycle AV program for cell cycle analysis (Phoenix Flow Systems, San Diego, CA, USA).

\section{Morphological assessment of apoptotic cells}

To detect apoptotic cells, SK-MES-1 cells were stained with the DNA-binding dye Hoechst 33258 (Dojindo, Kumamoto, Japan). After the cells were exposed to CrTX for $48 \mathrm{~h}$, they were fixed with $2 \%$ paraformaldehyde in PBS ( $\mathrm{pH} 7.4)$ for $10 \mathrm{~min}$ at $4^{\circ} \mathrm{C}$ and were then washed with PBS. To stain the nuclei, the cells were incubated for $10 \mathrm{~min}$ with $20 \mathrm{mg} / \mathrm{mL}$ Hoechst 33258. After washing with PBS, the cells were observed using an Olympus Microscope with a WU excitation filter (Tokyo, Japan).

\section{Western blot analysis}

SK-MES-1 cells were treated with $50 \mu \mathrm{g} / \mathrm{mL}$ CrTX for 24, 48, or $72 \mathrm{~h}$. Both adherent and floating cells were collected and frozen at $-80^{\circ} \mathrm{C}$. The protein expression levels of PCNA, caspase-3, LC3, p62, Beclin 1, p-p38, and p38 in SK-MES-1 cells were detected by Western blot analysis. Briefly, the cell pellets were resuspended in lysis buffer and then lysed at $4^{\circ} \mathrm{C}$ for $1 \mathrm{~h}$. After centrifugation at $12000 \times g$ for $10 \mathrm{~min}$, the protein content of the supernatant was determined using the Bio-Rad protein assay reagent (Bio-Rad, California, USA). Equal amounts of protein lysates $(50 \mu \mathrm{g})$ were separated by electrophoresis on $12 \%$ SDS-polyacrylamide gels and blotted onto nitrocellulose membranes. Protein expression was detected by rabbit polyclonal anti-PCNA, caspase-3, LC3, p62, Beclin 1, p-p38, and p38 antibodies (Santa Cruz Biotechnology, Santa Cruz, CA, USA) and a secondary antibody conjugated with peroxidase (goat anti-rabbit IgG) (Santa Cruz Biotechnology, Santa Cruz, CA, USA). The immunoreactive proteins on the membrane were visualized with an enhanced chemiluminescence detection system (Applygen Technologies Inc, Beijing, China). The images were scanned and analyzed semi-quantitatively using SigmaScan Pro 5 software (SPSS Inc, Chicago, IL, USA). The experimental values were normalized to $\beta$-actin reactivity.

\section{Statistical analysis}

All data are presented as the mean \pm standard deviation (SD). The differences between groups were evaluated using oneway ANOVA. The mean values were considered to be statistically significant at $P$ value $<0.05$.

\section{Results}

\section{CrTX inhibited the proliferation of SK-MES-1 cells}

The cytotoxic activity of CrTX was evaluated using the MTT assay. As shown in Figure 1, the cytotoxicity of CrTX in SKMES-1 cells was dose dependent. When SK-MES-1 cells were treated for $72 \mathrm{~h}$, the $\mathrm{IC}_{50}$ of CrTX was $25.13 \mu \mathrm{g} / \mathrm{mL}$.

\section{CrTX inhibited the colony formation of SK-MES-1 cells}

The colony formation assay provides an assessment of tumor cells' susceptibility to CrTX in an anchorage-independent environment. CrTX significantly diminished the capacity of

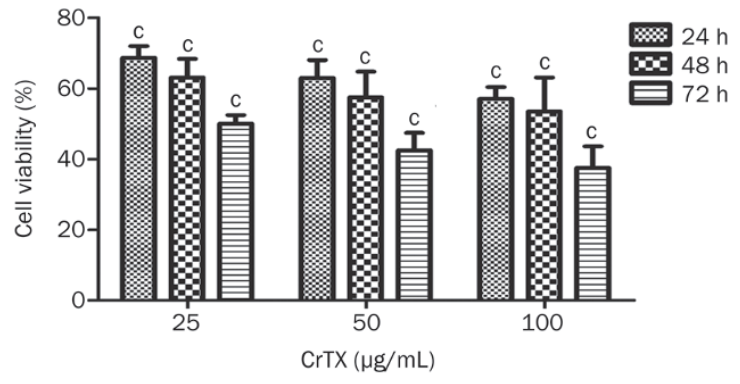

Figure 1. Dose- and time-dependent cytotoxicity of CrTX in SK-MES-1 cells. Cells were treated with various doses of CrTX, for 24,48 , or $72 \mathrm{~h}$. Values are from three independent experiments. $n=3$. Mean \pm SD. ${ }^{c} P<0.01$ vs control.

SK-MES-1 cells to form colonies. Compared with the control group, CrTX led to a $58 \%$ to $78 \%$ reduction in the number of colonies formed by SK-MES-1 cells $(P<0.01$, Figure 2$)$. These results suggest that $\mathrm{CrTX}$ can dramatically inhibit the viability of SK-MES-1 cells in vitro.
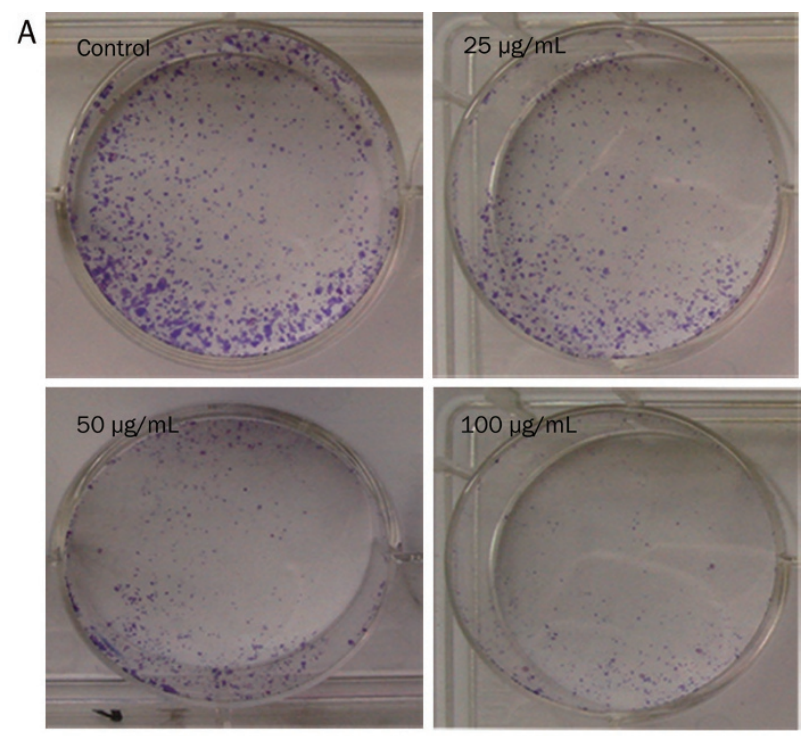

B

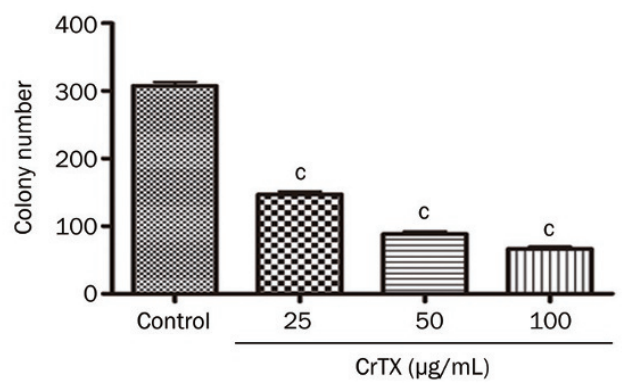

Figure 2. CrTX inhibited colony formation of SK-MES-1 cells. (A) Cells were seeded at 500 cells per plate, treated with/without CrTX, and allowed to form colonies. Colony numbers were counted and recorded. (B) Quantification of colony formation. There was a significant decrease in colony numbers of cells treated with CrTX compared with controls. $n=3$. Mean \pm SD. ${ }^{\circ} P<0.01$ vs control. 

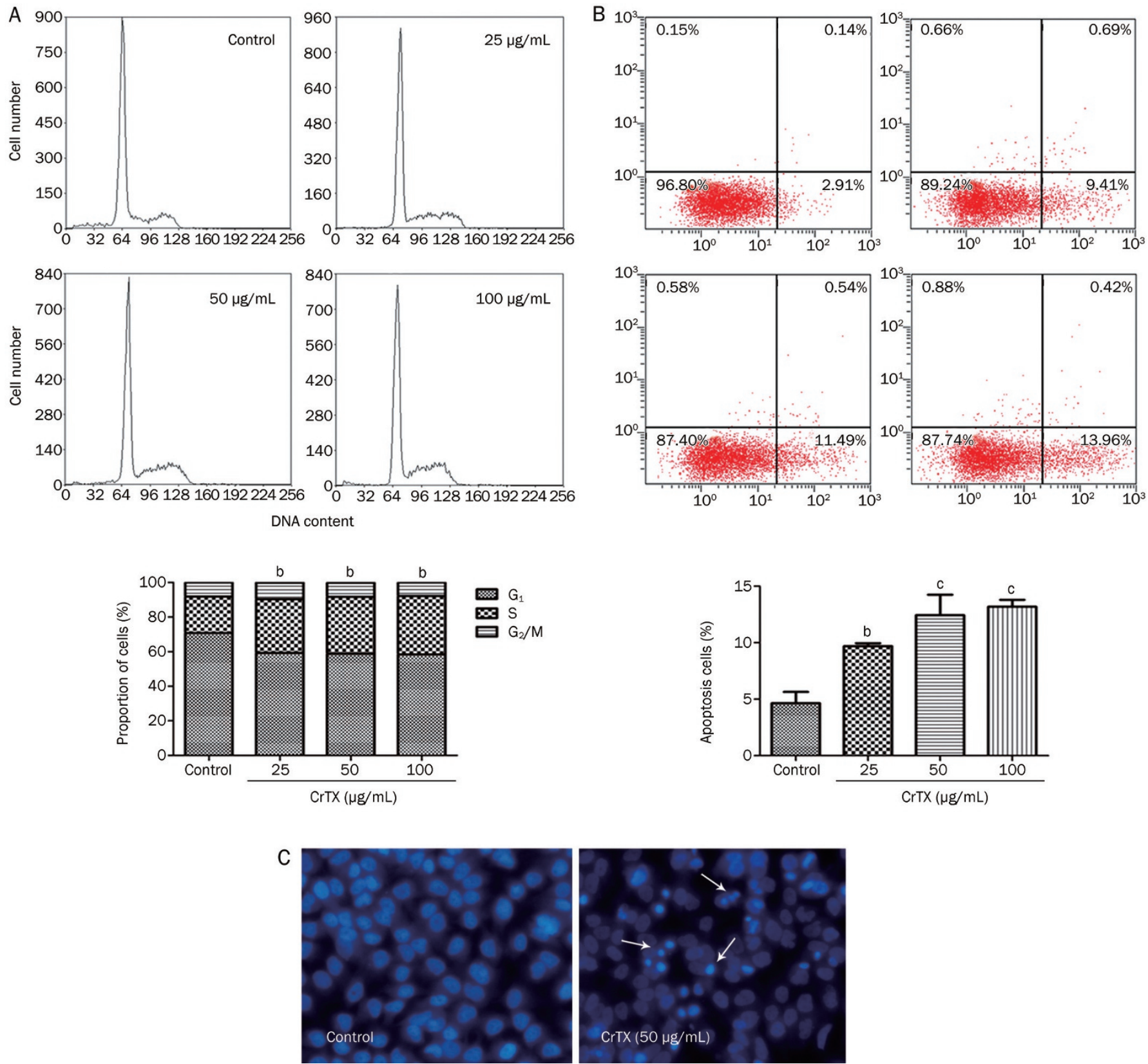

Figure 3. Effects of CrTX on cell cycle and apoptosis of SK-MES-1 cells. (A) SK-MES-1 cells were seeded into 50-mL culture flasks and incubated with 0, 25, and $50 \mu \mathrm{g} / \mathrm{mL}$, and $100 \mu \mathrm{g} / \mathrm{mL}$ CrTX for $48 \mathrm{~h}$. At the end of the incubation period, cells were harvested and stained with PI. Cell cycle was analyzed by flow cytometry. (B) The percentage of apoptotic cells was measured by flow cytometry. (C) After treatment with CrTX for $48 \mathrm{~h}$, cells were stained with Hoechst 33342 and morphology of apoptotic cells was observed under a fluorescence microscope (original magnification, $\times 200,0$ lympus, Tokyo, Japan). Condensed nuclei indicated cells underwent apoptosis.

CrTX inhibited the cell cycle and promoted the apoptosis of SKMES-1

The analysis of cell cycle kinetics and the quantitative detection of apoptotic cells were performed with flow cytometry. After treatment with CrTX at 25, 50, and $100 \mu \mathrm{g} / \mathrm{mL}$ for $48 \mathrm{~h}$, the proportion of cells in $\mathrm{G}_{0} / \mathrm{G}_{1}$ phase decreased, but the proportion of $S$ phase cells increased (Figure $3 \mathrm{~A}$ ). The proportions of apoptotic cells were $9.70 \%, 12.44 \%$, and $13.18 \%$, respectively, while the proportion of apoptotic cells was only $4.65 \%$ in the control group (Figure 3B). The morphological observation of SK-MES-1 cells showed that control SK-MES-1 cells had regular and round-shaped nuclei as revealed by nuclear staining (Figure 3C). By contrast, the condensation and fragmentation of the nuclei, characteristic of apoptotic cells, were observed in SK-MES-1 cells treated with CrTX. These results showed that treatment with CrTX induced a dose-dependent increase in the rate of apoptosis and $S$ phase cell cycle arrest in SK-MES-1 cells. 
Effects of CrTX on cell cycle and apoptotic proteins

PCNA (proliferating cell nuclear antigen) is a protein required for the function of DNA polymerase $\delta$ in eukaryotic cells. It is central to both DNA replication and repair. It follows that the level of PCNA expression may reflect the activity of cell proliferation. To further examine the effects of CrTX on the expression of apoptotic and cell cycle proteins, protein levels of PCNA and caspase-3 were evaluated using Western blot analysis. Figure 4 shows that caspase- 3 protein levels were increased after $48 \mathrm{~h}$ of treatment with CrTX, while PCNA protein levels decreased.

A
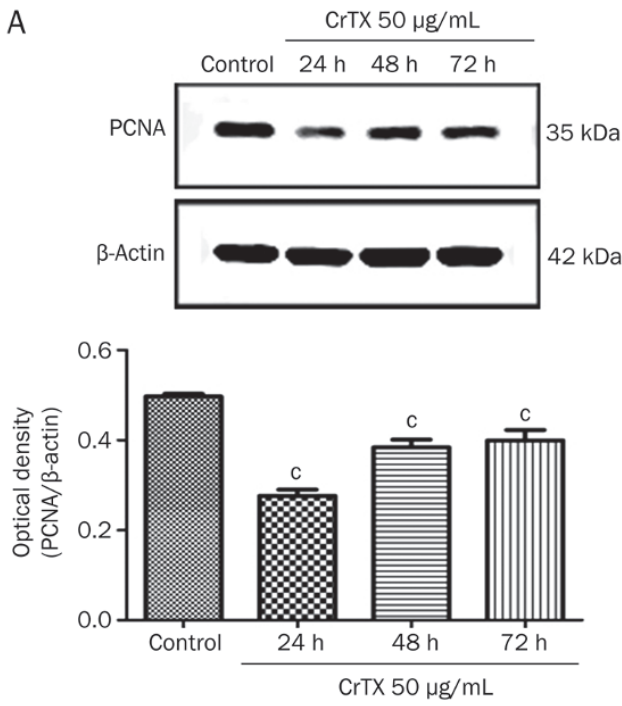

B
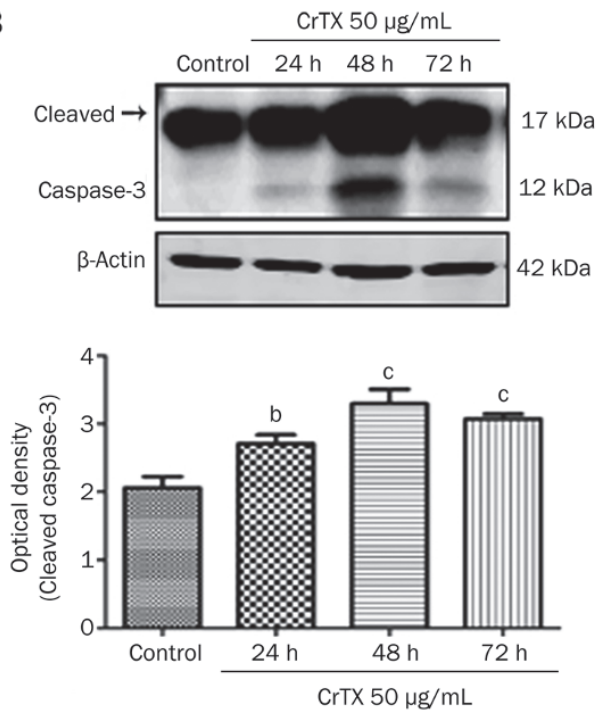

Figure 4. Effect of CrTX on the expressions of PCNA and cleaved caspase-3 in SK-MES-1 cells. SK-MES- 1 cells were treated with $50 \mu \mathrm{g} / \mathrm{mL}$ CrTX for 24,48 , and $72 \mathrm{~h}$. Cell lysates were prepared and protein levels of PCNA and caspase-3 were determined with Western blot analysis. (A) The expression of PCNA and quantification. (B) The expression of cleaved caspase-3 and quantification. $\beta$-Actin was used for normalization and verification of protein loading. $n=3$. Mean \pm SD. ${ }^{b} P<0.05,{ }^{c} P<0.01$ vs controls.

\section{Effects of CrTX on autophagy}

To evaluate if autophagy is activated in SK-MES-1 cells by CrTX, the levels of autophagy regulatory proteins (LC3, p62, and Beclin 1) were determined. The data revealed the up-regulation of the autophagy pathway following CrTX treatment evidenced by elevated levels of LC3-II and Beclin 1 proteins and the decreased protein expression of p62 (Figure 5).

P38MAPK is involved in CrTX-induced cytotoxicity, apoptosis and autophagy

To observe whether p38MAPK plays a role in the inhibition of SK-MES-1 cell growth by CrTX, protein levels of p-p38 and p38 were determined with Western blotting analysis. The results showed that after 24,48 , and $72 \mathrm{~h}$ of treatment with CrTX, p-p38 protein levels increased compared with the control group, while p38 protein levels remained unchanged (Figure 6). To further confirm the role of the p38 pathway, SB203580, a selective inhibitor of p38 MAPK, was used. The results showed that the CrTX-induced inhibition of cell proliferation, the increase in the rate of apoptosis and the elevation of cleaved caspase-3 expression were decreased after treatment with SB203580 (Figure 8). Similarly, the CrTX-induced upregulation of LC3-II and Beclin 1 protein and down-regulation of p62 protein levels were partially blocked by treatment with SB203580 (Figure 9). However, SB203580 pretreatment had no influence on CrTX-induced S phase arrest (Figure 7). These results indicated that $\mathrm{p} 38 \mathrm{MAPK}$ is an important mediator of the apoptotic response and autophagy activation in response to CrTX.

\section{Discussion}

CrTX, the main toxin of South American rattlesnake (Crotalus durissus terrificus) venom, was the first snake venom protein to be purified and crystallized. Numerous studies in recent years have shown that CrTX has antitumor activity in vivo and in vitro and interferes with tumor growth. A study of the cytotoxic and antitumor activities of CrTX by the National Cancer Institute USA (Developmental Therapeutics Program, NSC 624244) showed that CrTX was effective against human melanomas, central nervous system tumors and lung carcinomas. In 1995, the Food and Drug Administration (FDA) approved a phase I study of CrTX in patients with solid tumors refractory to conventional therapy ${ }^{[5]}$.

Previous studies from our group have shown that CrTX can enhance the antitumor effect of tyrosine kinase inhibitors in SK-MES-1 cells with acquired resistance mediated by the JNK signal transduction pathway ${ }^{[25]}$. In this study, we used the MTT and colony formation assays to further demonstrate that CrTX induces a cytotoxic effect. The results confirm that CrTX effectively suppressed the proliferation and colony formation of SK-MES-1 cells in vitro in a dose- and time-dependent manner. Furthermore, we found that CrTX induced S phase cell cycle arrest in SK-MES-1 cells. These results suggest that CrTX suppresses the proliferation of cells through the inhibition of DNA synthesis followed by the induction of S-phase arrest, and this arrest correlated with a decrease in the levels 
A

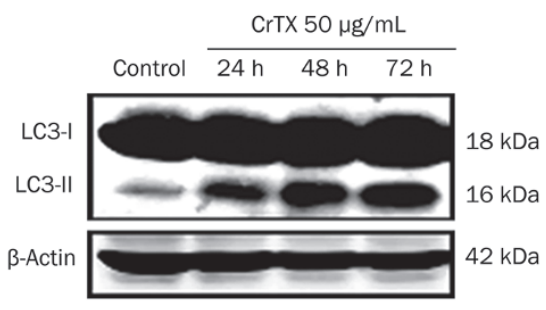

B

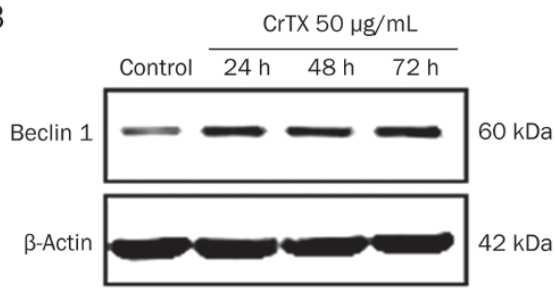

C

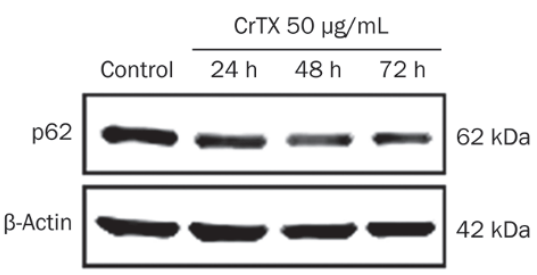

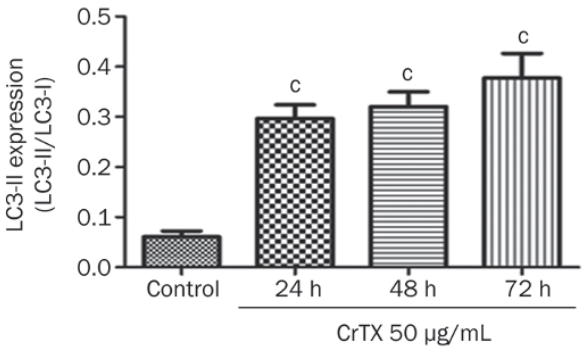
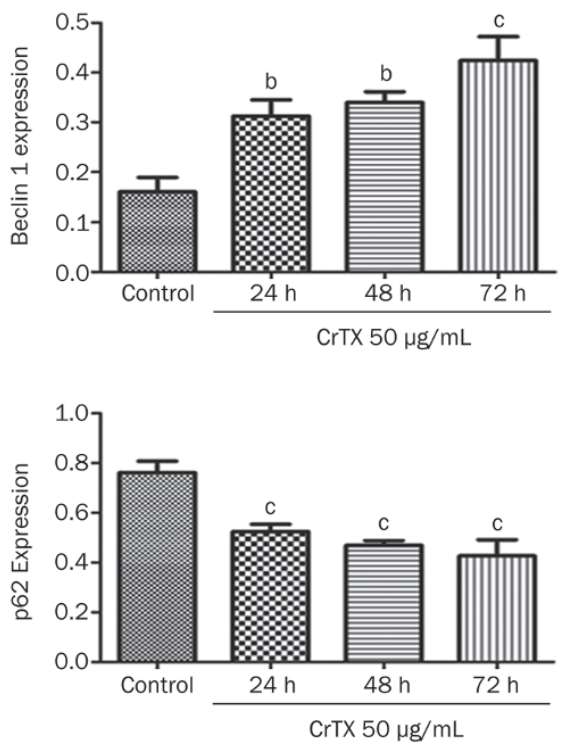

Figure 5. Effects of CrTX on the expression of LC3, Beclin 1, and p62 in SK-MES-1 cells. SK-MES-1 cells were treated with $50 \mu \mathrm{g} / \mathrm{mL}$ CrTX for 24,48 , and $72 \mathrm{~h}$. Cell lysates were prepared and protein levels of LC3, Beclin 1, and p62 were determined with Western blot analysis. (A) The expression of LC3 and quantification. (B) The expression of Beclin 1 and quantification. (C) The expression of p62 and quantification. $\beta$-Actin was used for normalization and verification of protein loading. $n=3$. Mean \pm SD. ${ }^{b} P<0.05,{ }^{c} P<0.01$ vs controls.

of PCNA.

The induction of apoptosis in target cells is a key mechanism for most antitumor therapies. Caspases, a family of proteases, play a central role in apoptotic mechanism. There are three pathways to activate caspases: the intrinsic, extrinsic and intrinsic endoplasmic reticulum pathways. Caspase-9 is upstream of the intrinsic pathway, and caspase- 8 is upstream of the extrinsic pathway. The intrinsic and extrinsic pathways converge to caspase-3; therefore, caspase- 3 is considered to be a key enzyme in the pathogenesis of cell apoptosis. When the full-length pro-caspase- $3(32 \mathrm{kDa})$ is activated, it is cleaved to form two mature subunits: p17 $(17 \mathrm{kDa})$ and p12 $(12 \mathrm{kDa})$. The level of the cleaved caspase- 3 represents the level of activated caspase- $-3^{[26,27]}$. Our results show that CrTX increases the proportions of apoptotic cells. Additionally, it was observed by nuclear staining with Hoechst 33258 that SK-MES-1 cells treated with CrTX showed condensation and fragmentation of nuclei, which are characteristics of apoptotic cells. Furthermore, we examined the expression of caspase-3 protein using Western blot analysis. The result showed that the level of cleaved caspase- 3 was increased. These studies suggest that CrTX treatment induces apoptosis in SK-MES-1 cells.
Autophagy is a mechanism by which cells enhance the metabolism of damaged organelles or recycle dispensable materials to survive harsh conditions. The role of autophagy in cancer is dual-sided. The loss of autophagy may increase the propensity of cells toward oncogenic transformation ${ }^{[28]}$. In this case, the induction of autophagy would seem to be beneficial for cancer prevention. In established tumors, however, autophagy can be tumor-promoting, and cancer cells can use enhanced autophagy to survive under metabolic and therapeutic stress ${ }^{[29]}$. Thus, the rational modulation of autophagy based on its functional status in tumors is very important in antitumor therapy. LC3 is one of the members of the Atg family that is implicated in the formation of the autophagosomes. The cleavage of LC3 at the carboxy terminus immediately following synthesis yields the cytosolic LC3-I form. In the initiation of autophagy, LC3 (type I) can be lipidated to the active form (type II), which can then interact with cellular lipids to facilitate the aggregation of autophagosomes ${ }^{[30,31]}$. The conversion of LC3-I to LC3-II has been used as a marker for autophagy. Beclin 1 plays an essential role in the initiation of autophagy, as autophagosome nucleation requires Beclin $1^{[32]}$. P62 is a substrate of autophagy. A decrease in levels of 
A
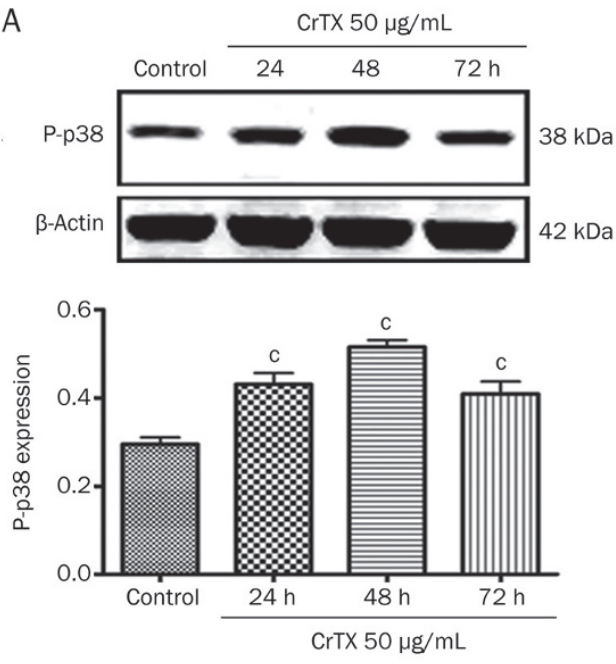

B
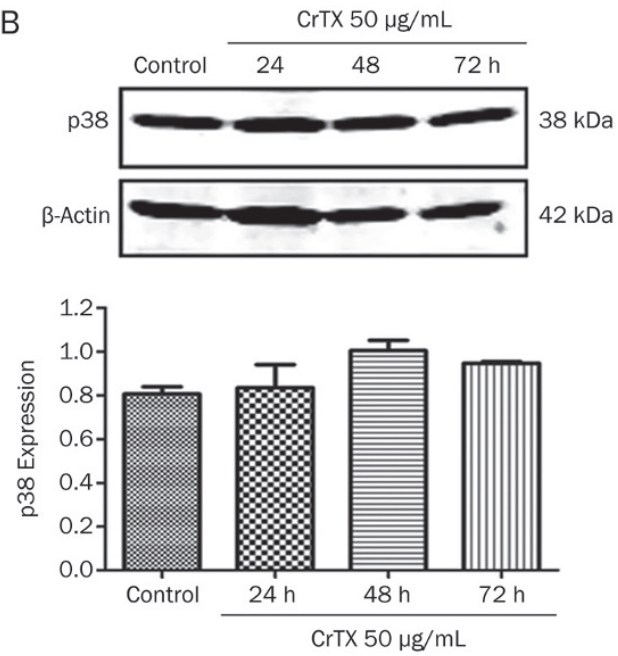

C

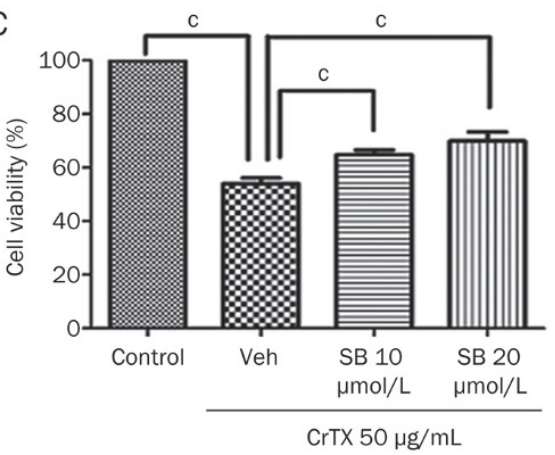

Figure 6. Effects of p38MAPK on CrTX-induced cytotoxicity in SK-MES-1 cells. SK-MES-1 cells were treated with $50 \mu \mathrm{g} / \mathrm{mL}$ CrTX for 24,48 , and $72 \mathrm{~h}$. Cell lysates were prepared and protein levels of p-p38 and p38 were determined by Western blotting analysis. SK-MES-1 cells were pretreated with or without SB (10 or $20 \mu \mathrm{mol} / \mathrm{L}, 1 \mathrm{~h}$ ), followed by 50 $\mu \mathrm{g} / \mathrm{mL}$ of $\mathrm{CrTX}$ for $48 \mathrm{~h}$, and then cell viability was determined by MTT assay. (A) The expression of p-p38 and quantification after CrTX induction at indicated time. $\beta$-Actin was used for normalization and verification of protein loading. (B) The expression of $p 38$ and quantification. (C) Effect of SB203580 on the cytotoxicity of CrTX. $n=3$. Mean \pm SD. ${ }^{b} P<0.05,{ }^{c} P<0.01$ vs controls. p62 is observed when autophagy activity is increased. In contrast, an increase in p62 levels may be caused by a decrease in autophagy activity. Thus, levels of p62 can be used as an index of autophagy activity ${ }^{[33]}$. It has been reported that CrTX enhanced autophagy in MCF-7 cells by activating cathepsin $\mathrm{B}, \mathrm{D}$, and L, releasing cytochrome $c$, and relocating AIF into nuclei ${ }^{[13]}$. Similar results were observed in this study; CrTX elevated the expression of both LC3-II and Beclin 1 protein and decreased $\mathrm{p} 62$ protein levels, suggesting that autophagy may be involved in the inhibitory effects of CrTX on SK-MES-1 cells.

p38MAPK signaling pathways have been demonstrated to play a central role in diverse biological processes, such as cell proliferation, apoptosis and autophagy ${ }^{[34-36,22]}$. Recent studies have shown that p38MAPK pathways are activated in response to various stresses and chemicals. Wang et al reported that $\mathrm{p} 38 \mathrm{MAPK}$ regulated caspase-3 activity by binding to caspase- 3 in the nucleus of Bel-7402 cells during antiFas antibody- and actinomycin D-induced apoptosis ${ }^{[37]}$. In this study, we examined the $\mathrm{p} 38$ and $\mathrm{p}-\mathrm{p} 38$ proteins to elucidate the potential effects of CrTX on p38 activation in SK-MES-1 cells. The results showed that the levels of p-p38 were significantly increased by CrTX, and the cytotoxicity, apoptosis and autophagy induced by CrTX in SK-MES-1 cells were decreased after treatment with the selective p38 inhibitor SB203580. However, SB203580 pretreatment had no influence on CrTX-induced S phase arrest, suggesting that p38MAPK is not involved in the induction of $S$ phase arrest. CrTX may be associated with other pathways, such as DNA damage and replication, and its mechanism needs to be further explored. Therefore, our results suggest that p38MAPK signaling is at least partially responsible for CrTX-induced apoptosis and autophagy in SK-MES-1 cells.

In conclusion, our results indicate that CrTX inhibits the proliferation of SK-MES-1 cells by inducing S phase arrest, apoptosis and autophagy. Additionally, we demonstrated that the p38MAPK signal transduction pathway is activated by CrTX and mediates CrTX-induced apoptosis and autophagy activation. These results will help us understand the molecular basis of CrTX-induced cell death in lung cancer and shed new light on development of CrTX-based cancer therapeutics.

\section{Acknowledgements}

This study was funded by the project of the Priority Academic Program Development of Jiangsu Higher Education Institutions (PAPD) and Suzhou Science and Technology Plan Projects in 2012 (№ SYS201205).

\section{Author contribution}

Chun-yu LIU and Zheng-hong QIN designed and directed the study. Rong HAN drafted the manuscript. Hui LIANG performed most of the experiments and data analysis. Rong HAN participated in the study design and coordination. All authors read and approved the final manuscript. 

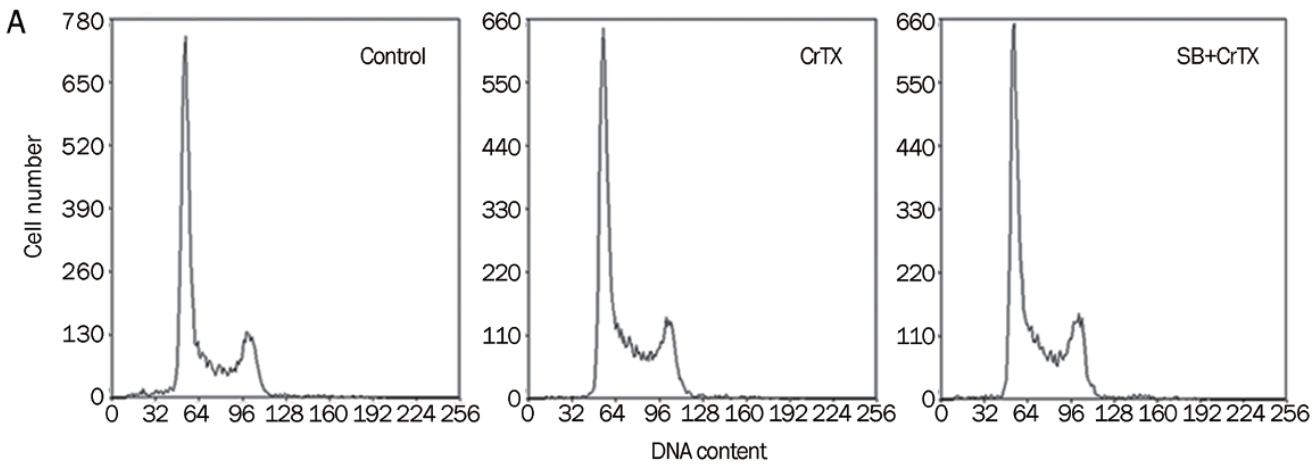

B

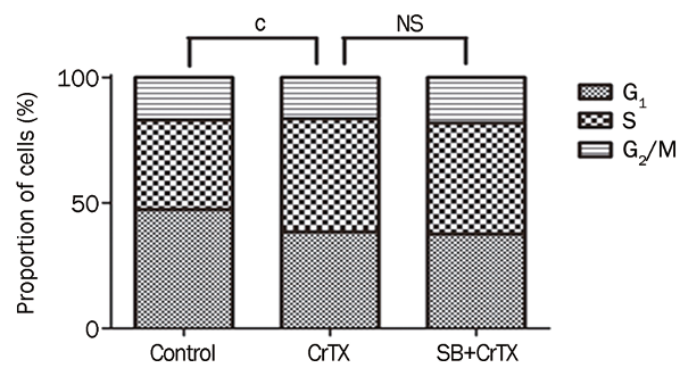

Figure 7. Effects of SB on CrTX-induced S arrest of SK-MES-1 cells. (A) SK-MES-1 cells were pretreated with or without SB (10 $\mu$ mol/L, 1 h), followed by $50 \mu \mathrm{g} / \mathrm{mL}$ of CrTX for $48 \mathrm{~h}$, and then cell cycle was analyzed with FCM. (B) Statistical analysis. $n=3$. Mean \pm SD. ${ }^{b} P<0.05,{ }^{c} P<0.01$ vs controls.
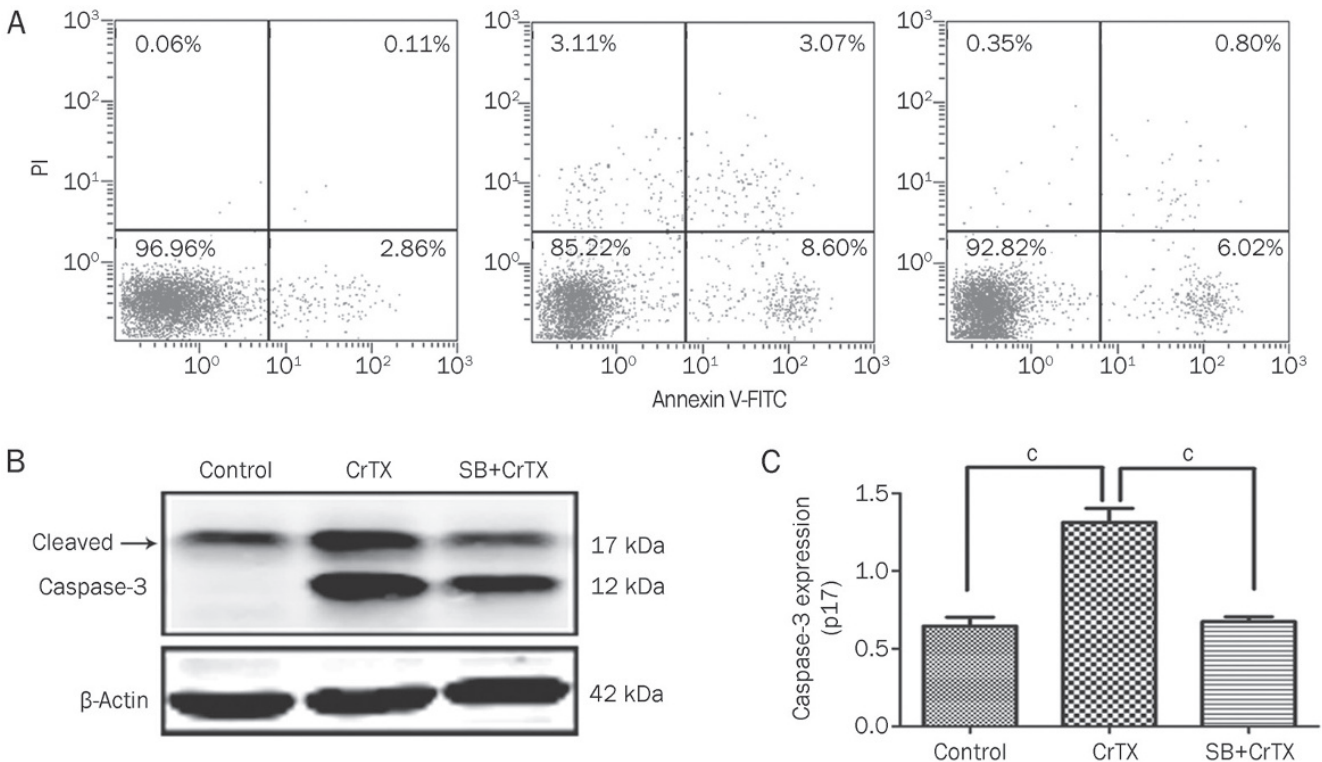

Figure 8. Effect of SB on CrTX-induced apoptosis of SK-MES-1 cells. SK-MES-1 cells were pretreated with or without SB (10 $\mu$ mol/L, $1 \mathrm{~h}$ ), followed by $50 \mathrm{\mu g} / \mathrm{mL}$ of CrTX for $48 \mathrm{~h}$. (A) Apoptosis of SK-MES-1 cells. Cells were collected and stained with Annexin V-FITC/PI, and then subjected to flow cytometry to detect apoptotic cells of SK-MES-1 cells. (B) The expression of cleaved caspase-3 was detected by Western blot analysis. $\beta$-Actin was used for normalization and verification of protein loading. $n=3$. Mean \pm SD. ${ }^{b} P<0.05,{ }^{c} P<0.01$ vs controls.

\section{References}

1 Jemal A, Siegel R, Xu J, Ward E. Cancer statistics. CA Cancer J Clin 2010; 60: 277-300.

2 Thanyanan R, Saravut JW, Julian RM. Oncogenic pathways, molecularly targeted therapies, and highlighted clinical trials in nonsmall-cell lung cancer (NSCLC). Clin Lung Cancer 2012; 13: 252-66.
3 Pereańez JA, Gómez ID, Patińo AC. Relationship between the structure and the enzymatic activity of crotoxin complex and its phospholipase A2 subunit: an in silico approach. J Mol Graph Model 2012; 35: $36-42$.

4 Faure G, Xu H, Saul FA. Crystal structure of crotoxin reveals key residues involved in the stability and toxicity of this potent hetero- 
A

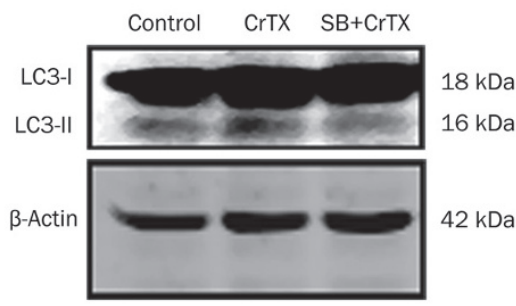

B

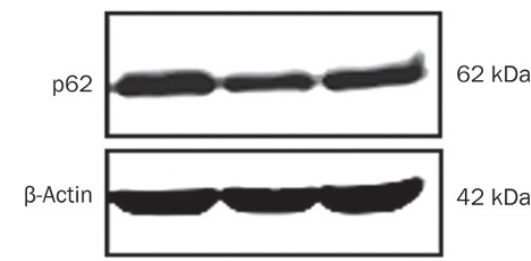

C

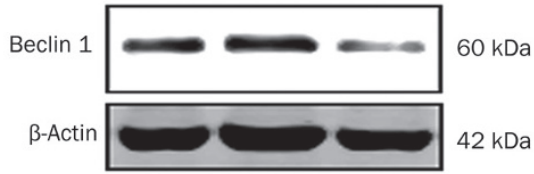

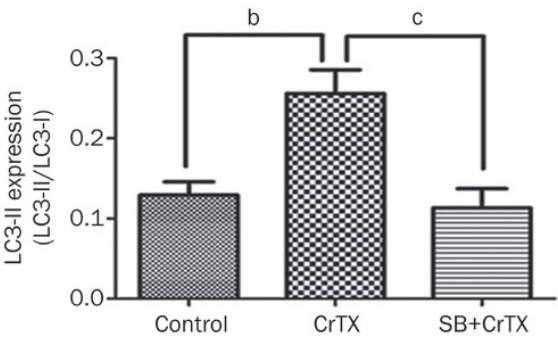
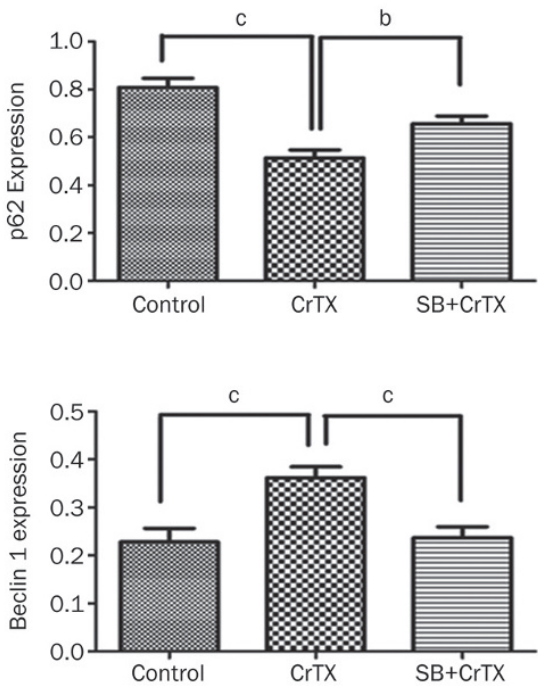

Figure 9. Effects of SB on LC3 (A), p62 (B), and Beclin 1 (C) protein levels in SK-MES-1 cells. SK-MES-1 cells were pretreated with or without SB (10 $\mu \mathrm{mol} / \mathrm{L}, 1 \mathrm{~h}$ ), followed by $50 \mu \mathrm{g} / \mathrm{mL}$ of CrTX for $48 \mathrm{~h}$. The expressions of LC3, p62, and Beclin 1 were detected by Western blot analysis. $\beta$-Actin was used for normalization and verification of protein loading. $n=3$. Mean \pm SD. ${ }^{b} P<0.05,{ }^{c} P<0.01$ vs controls.

dimeric ß-neurotoxin. J Mol Biol 2011; 412: 176-91.

5 Sampaio SC, Hyslop S, Fontes MR, Prado-Franceschi J, Zambelli Vo, Magro AJ, et al. Crotoxin: novel activities for a classic beta-neurotoxin. Toxicon 2010; 55: 1045-60.

6 Baldi A, Mordoh J, Medrano EE, de Bonaparte YP, de Lustig ES, Rumi L. Special report: studies to determine the possible antitumoral properties of cobra venom and crotoxin complex A and B. Medicina (B Aires) 1988; 48: 337-44.

7 Corin RE, Viskatis LJ, Vidal JC, Etcheverry MA. Cytotoxicity of crotoxin on murine erythroleukemia cells in vitro. Invest New Drugs 1993; 11: $11-5$.

8 Newman RA, Vidal JC, Viskatis LJ, Johnson J, Etcheverry MA. VRCTC-310, a novel compound of purified animal toxin separates antitumor efficacy from neurotoxicity. Invest New Drugs 1993; 11 : 151-9.

9 Rudd CJ, Viskatis $\sqcup$, Vidal JC, Etcheverry MA. In vitro comparison of cytotoxic effects of crotoxin against three human tumors and a normal human epidermal keratinocyte cell line. Invest New Drugs 1994; 12: 183-4.

10 Donato NJ, Martin CA, Perez M, Newman RA, Vidal JC, Etcheverry MA. Regulation of epidermal growth factor receptor activity by crotoxin, a snake venom phospholipase A2 toxin. A novel growth inhibitory mechanism. Biochem Pharmacol 1996; 51: 1535-43.

11 Cura JE, Blanzaco DP, Brisson C, Cura MA, Cabrol R, Larrateguy L, et al. Phase I and pharmacokinetics study of crotoxin (cytotoxic PLA2, NSC-624244) in patients with advanced cancer. Clin Cancer Res 2002; 8: 1033-41.
12 Yan CH, Liang ZQ, Gu ZL, Yang YP, Reid P, Qin ZH. Contributions of autophagic and apoptotic mechanisms to CrTX-induced death of K562 cells. Toxicon 2006; 47: 521-30.

13 He JK, Wu XS, Wang Y, Han R, Qin ZH, Xie Y. Growth inhibitory effects and molecular mechanisms of crotoxin treatment in esophageal Eca109 cells and transplanted tumors in nude mice. Acta Pharmacol Sin 2012; 34: 295-300.

14 Johnson GL, Lapadat R. Mitogen-activated protein kinase pathways mediated by ERK, JNK, and p38 protein kinases. Science 2002; 298: 1911-2.

15 Junttila MR, Li SP, Westermarck J. Phosphatase-mediated crosstalk between MAPK signaling pathways in the regulation of cell survival. FASEB J 2008; 22: 954-65.

16 Zarubin T, Han J. Activation and signaling of the p38 MAP kinase pathway. Cell Res 2005; 15: 11-8.

17 Wagner EF, Nebreda AR. Signal integration by JNK and p38 MAPK pathways in cancer development. Nat Rev Cancer 2009; 9: 537-49.

18 Zhan Y, Gong K, Chen C, Wang H, Li W. P38 MAP kinase functions as a switch in MS-275-induced reactive oxygen species-dependent autophagy and apoptosis in human colon cancer cells. Free Radic Biol Med 2012; 53: 532-43.

19 de la Cruz-Morcillo MA, Valero ML, Callejas-Valera JL, Arias-González L, Melgar-Rojas P, Galán-Moya EM, et al. P38MAPK is a major determinant of the balance between apoptosis and autophagy triggered by 5-fluorouracil: implication in resistance. Oncogene 2012; 31: 107385.

20 Wang Y, Sun LG, Xia CH, Ye LP, Wang B. P38MAPK regulates 
caspase-3 by binding to caspase-3 in nucleus of human hepatoma Bel-7402 cells during anti-Fas antibody- and actinomycin D-induced apoptosis. Biomed Pharmacother 2009; 63: 343-50.

21 Bradham C, McClay DR. p38 MAPK in development and cancer. Cell Cycle 2006; 5: 824-8.

22 Choi CH, Jung YK, Oh SH. Autophagy induction by capsaicin in malignant human breast cells is modulated by p38 and extracellular signal-regulated mitogen-activated protein kinases and retards cell death by suppressing endoplasmic reticulum stress-mediated apoptosis. Mol Pharmacol 2010; 78: 114-25.

23 Neoh CA, Wang RY, Din ZH, Su JH, Chen YK, Tsai FJ, et al. Induction of apoptosis by sinulariolide from soft coral through mitochondrialrelated and p38 MAPK pathways on human bladder carcinoma cells. Mar Drugs 2012; 10: 2893-911.

24 Ye B, Xie Y, Qin ZH, Wu JC, Han R, He JK. Anti-tumor activity of CrTX in human lung adenocarcinoma cell line A549. Acta Pharmacol Sin 2011; 32: $1397-401$.

25 Wang JH, Xie Y, Wu JC, Han R, Reid PF, Qin ZH, et al. Crotoxin enhances the antitumor activity of gefinitib (Iressa) in SK-MES-1 human lung squamous carcinoma cells. Oncol Rep 2012; 27: 13417.

26 Chaabane W, User SD, El-Gazzah M, Jaksik R, Sajjadi E, RzeszowskaWolny J, et al. Autophagy, apoptosis, mitoptosis and necrosis: interdependence between those pathways and effects on cancer. Arch Immunol Ther Exp (Warsz) 2013; 61: 43-58.

27 Wong RS. Apoptosis in cancer: from pathogenesis to treatment. J Exp Clin Cancer Res 2011; 30: 1-14.

28 Lozy F, Karantza V. Autophagy and cancer cell metabolism. Semin
Cell Dev Biol 2012; 23: 395-401.

29 Choi KS. Autophagy and cancer. Exp Mol Med 2012; 44: 109-20.

30 Mizushima N, Levine B. Autophagy in mammalian development and differentiation. Nat Cell Biol 2010; 12: 823-30.

31 Mizushima N. Autophagy: process and function. Genes Dev 2007; 21: 2861-73.

32 Proikas-Cezanne T, Codogno P. Beclin 1 or not Beclin 1. Autophagy 2011; 7: 671-2.

33 Bjørkøy G, Lamark T, Pankiv S, Øvervatn A, Brech A, Johansen T. Monitoring autophagic degradation of p62/SQSTM1. Methods Enzymol 2009; 452: 181-97.

34 Pedersen IM, Buhl AM, Klausen P, Geisler CH, Jurlander J. The chimeric anti-CD20 antibody rituximab induces apoptosis in B-cell chronic lymphocytic leukemia cells through a p38 mitogen activated protein-kinase-dependent mechanism. Blood 2002; 99: 1314-9.

35 Tang G, Yue Z, Talloczy Z, Hagemann T, Cho W, Messing A, et al. Autophagy induced by Alexander disease-mutant GFAP accumulation is regulated by $\mathrm{p} 38 / \mathrm{MAPK}$ and mTOR signaling pathways. Hum Mol Genet 2008; 17: 1540-55.

36 Liu B, Cheng Y, Zhang B, Bian HJ, Bao JK. Polygonatum cyrtonema lectin induces apoptosis and autophagy in human melanoma A375 cells through a mitochondria-mediated ROS-p38-p53 pathway. Cancer Lett 2009; 275: 54-60.

37 Wang Y, Sun LG, Xia CH, Ye LP, Wang B. P38MAPK regulates caspase- 3 by binding to caspase- 3 in nucleus of human hepatoma Bel-7402 cells during anti-Fas antibody- and actinomycin D-induced apoptosis. Biomed Pharmacother 2009; 63: 343-50. 